\title{
EFFECTS OF DISRUPTIVE SELECTION
}

\section{COUPLING AND REPULSION}

J. M. THODAY

Genetics Department, Sheffield University*

Received I8.ii.59

\section{INTRODUCTION}

IT has already been shown experimentally (Thoday, I958, I959) that disruptive selection (Mather, I953) or centrifugal selection (Simpson, I944) can increase and that stabilising (or centripetal) selection can reduce the genetic flexibility of a population. The effects of disruptive selection can also extend so far as to render a population polymorphic (Thoday and Boam, I959), as Mather (1955) had expected on general grounds.

These experiments involved selection for sternopleural chæta number in populations initiated from a wild stock of Drosophila melanogaster. They were open to interpretation in terms of Mather's (1943) models, on the supposition that disruptive selection can increase the frequency of coupling linkages of genes hitherto present mostly in repulsion complexes, and that stabilising selection can increase the frequency of repulsion complexes and, if populations are small, render them homozygous.

The experiments to be reported here complement those described before. They were initiated from a cross between two lines differing widely in chæta number, so that relevant genes could be assumed to have been in the coupling phase of linkage at the beginning of selection. They also supplement the previous experiments in that the lines crossed differed at four marker loci, two of which were known to be linked to a chromosome region with a strong effect on chaeta number. It was hoped that these markers would provide information about the parental or recombinant chromosomes that disruptive or stabilising selection might favour.

\section{TERMINOLOGY}

The terminology of selection is becoming confusing, and a restatement of the definitions to be used seems desirable. The terms stabilising and disruptive are used here in the sense defined in the first paper of this series (Thoday, I959) and are only intended to denote the kind of selection exercised by the experimenter. They are not used to describe the expected consequences of selection. These terms and others, however, are often used in relation to the expected consequences of selection. Waddington (I953), for example, felt it necessary to divide stabilising selection into normalising and stabilising according to

* Present address : Department of Genetics, 44 Storey's Way, Cambridge. 
whether the consequences were a reduction of genetic variance or an increase of developmental stability, and Lerner (1958, fig. I) has felt it necessary to describe disruptive selection as stabilising, presumably because disruptive selection may sometimes stabilise gene frequencies.

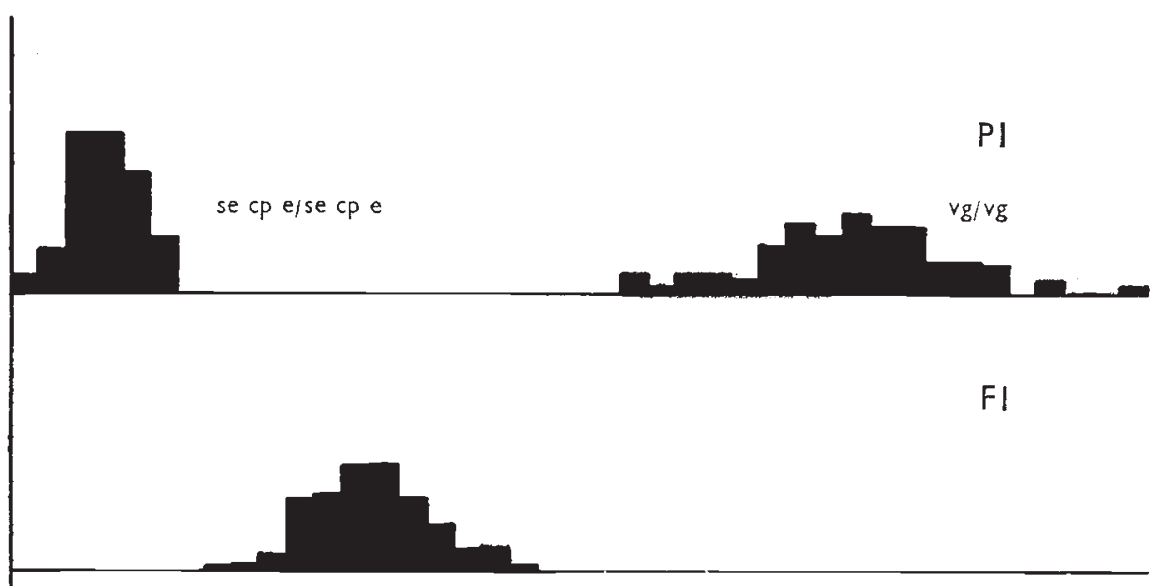

F2
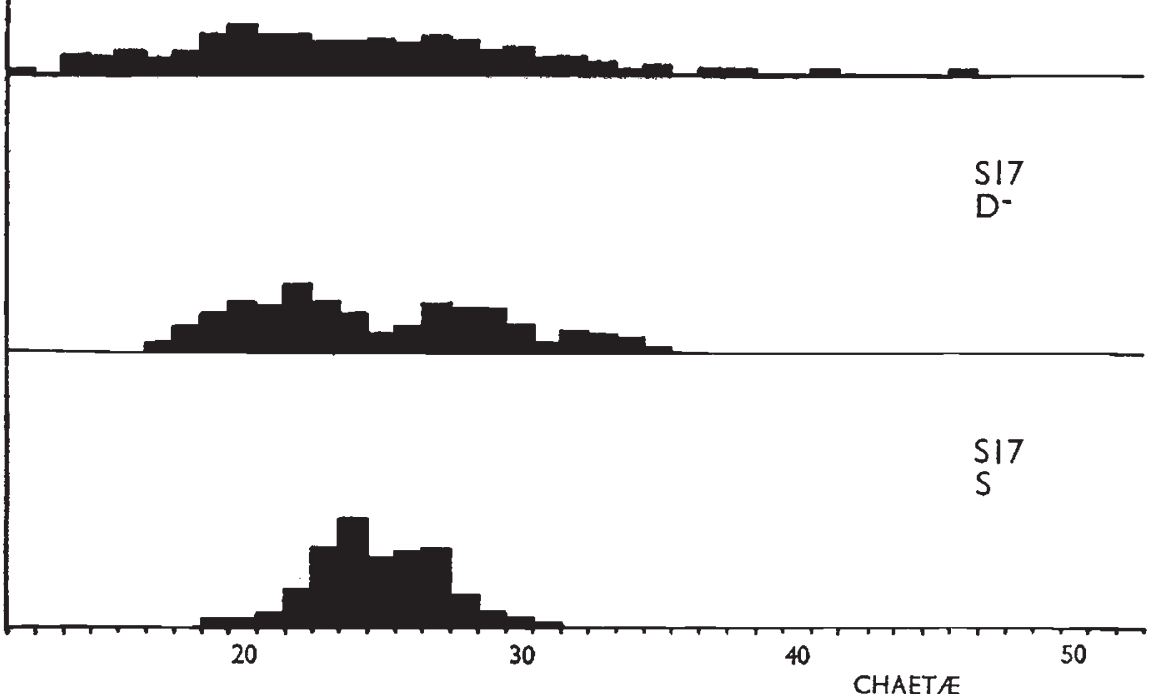

Fig. I.-Distributions of chæta number in $P_{1}, F_{1}, F_{2}$ and in the two lines in $S_{1} 7$. Each histogram is based on 160 flies except that for $F_{2}$ which is based on 320 .

In these circumstances it might be better to follow Falconer's (1958) use of Simpson's relatively neutral terms, centripetal and centrifugal selection. But it seems a pity to change terminology in the middle of a series of papers. The terms disruptive and stabilising 
will still be used, therefore, but the reader is reminded that they are used in senses independent of their expected consequences. Stabilising selection is selection for the mean. Disruptive selection is selection away from the mean in both directions in the same population.

Waddington (1958) has also suggested that disruptive selection can adequately be regarded as a subtype of directional selection. This is clearly illegitimate as the terms are used here, and is also unsatisfactory when the two do have different consequences.

\section{MATERIAL}

The experiments have involved the maintenance of two lines, one (S) under stabilising selection, the other $\left(\mathrm{D}^{-}\right)$under disruptive selection with negative assortative mating. These were both initiated from the same four $F_{1}$ single-pair cultures. The $\mathrm{F}_{1}$ was a cross between a selection line "vg 6 ", whose history is to be described elsewhere, and a stock homozygous for the third chromosome recessives $s e, c p$ and $e$.

The line vg 6 is homozygous for the second chromosome recessive $v g$ and, when the $F_{1}$ was made, had a mean sternopleural chaeta-number of 40 as a result of 72

TABLE I

Mean chaeta-numbers of $F_{2}$ fies classifed by vg and se phenotype

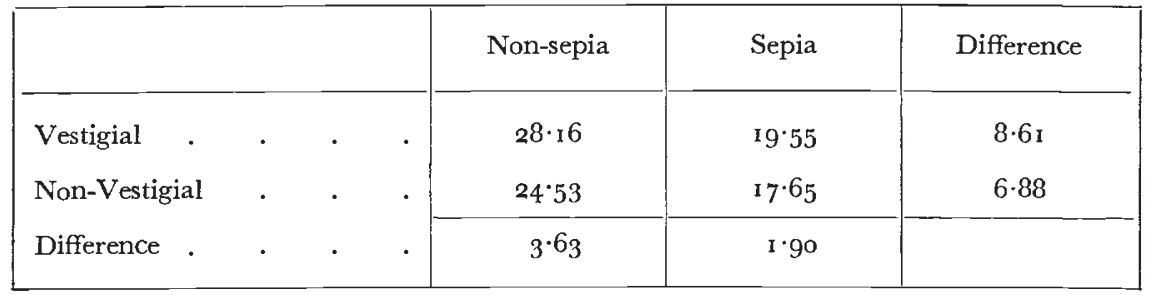

generations of selection for high chaeta number. The mean chaeta number of the se cpe stock was about 15 . The chaeta number frequency distribution curves for the two parent lines, their $\mathbf{F}_{\mathbf{1}}$ and $\mathbf{F}_{\mathbf{2}}$ are given in fig. $\mathbf{1}$, which illustrates the very large variance available at the start of the experiments.

It is clear from the $F_{2}$ data that chaeta number genes were linked both to the $v g$ and to the se loci. The vestigial winged flies averaged 25.5 chaetæ and the nonvestigial 23.2 chaetæ. The sepia-eyed flies averaged 18.3 chaetæ and the non-sepia 25.3 chaetæ. Chromosome III is the more important, but there is evidence of interaction between the chromosomes which is summarised in table 1 . The effect of the high third chromosomes was greater in $v g$ homozygotes.

Some test-crosses have been made to analyse the differences affecting chaeta number that distinguish the vg 6 chromosome III from more normal third chromosomes. These were done earlier in the history of $\mathrm{vg} 6$, well before $\mathrm{vg} 6$ was used to set up the present experiments. They indicated that flies heterozygous for the third chromosomes of vg 6 and the se cpe stock, differed from those homozygous for the se cp e chromosome by about 3 chaetæ, and that the right arm of the chromosomes did not contribute significantly to this difference, which was confined to regions to the left of the locus $p$. Some of the difference, probably the major part, is to the right of se but to the left of $c p$. Most of the difference is to the left of $r t$, but some of it may be to the right of rt. Marked chromosomes suitable for further analysis have yet to be synthesised, and at present the test-crosses have provided no certain information whether the third chromosomes differ at one or several 
loci that affect chaeta number. However, the experiments were initiated on the assumption that the relevant region of the vg 6 chromosome III contained a linked group of polygenes as Mather's (1943) ideas would imply. The results clearly agree with this assumption (see below).

\section{MAINTENANCE OF THE LINES}

Culture technique, assay, and selection were identical with those described for the $\mathrm{D}^{-}$and $\mathrm{S}$ lines of Thoday (1959), with the one exception that no preference for more bilaterally symmetrical flies was exercised in selection.

One hundred and sixty flies, 20 of each sex from each of four cultures were assayed in each line in each generation. Each culture was a single pair culture and, provided that the best single pair cultures were successful, selection involved choosing one fly out of each 20.

TABLE 2

Mating and selection system for $D^{-}$line

\begin{tabular}{|c|c|c|c|c|}
\hline \multirow{3}{*}{$\begin{array}{l}\text { Parents of } \\
\text { generation }\end{array}$} & \multicolumn{4}{|c|}{ Culture } \\
\hline & A & B & $\mathrm{C}$ & $\mathrm{D}$ \\
\hline & q $\quad \pi$ & q $\quad \pi$ & 웅 & q \\
\hline $\begin{array}{c}n \\
n+1 \\
n+2 \\
n+3 \\
\text { etc. }\end{array}$ & $\begin{array}{l}\mathrm{HA} \times \mathrm{LC} \\
\mathrm{HA} \times \mathrm{LD} \\
\mathrm{HA} \times \mathrm{LG} \\
\mathrm{HA} \times \mathrm{LD}\end{array}$ & $\begin{array}{l}\mathrm{HB} \times \mathrm{LD} \\
\mathrm{HB} \times \mathrm{LC} \\
\mathrm{HB} \times \mathrm{LD} \\
\mathrm{HB} \times \mathrm{LC}\end{array}$ & $\begin{array}{l}\mathrm{LC} \times \mathrm{HA} \\
\mathrm{LC} \times \mathrm{HB} \\
\mathrm{LC} \times \mathrm{HA} \\
\mathrm{LC} \times \mathrm{HB}\end{array}$ & $\begin{array}{l}\mathrm{LD} \times \mathrm{HB} \\
\mathrm{LD} \times \mathrm{HA} \\
\mathrm{LD} \times \mathrm{HB} \\
\mathrm{LD} \times \mathrm{HA}\end{array}$ \\
\hline
\end{tabular}

The entries designate the parents used to produce the culture in the generation shown in the first column. H indicates the highest, and $\mathrm{L}$ the lowest chaeta number fly found in the appropriate culture. A, B, C and D indicate the culture from which the fly was selected.

The $\mathrm{D}^{-}$(Disruptive selection with negative assortative mating) mating and selection system is reproduced in table 2. The $\mathrm{S}$ line mating system was identical, but selection in this line was aimed at the $\mathrm{F}_{1}$ mean value $(24 \cdot 5)$. The female with the nearest to 25 chaetæ and the male with the nearest to 24 chaetæ were chosen. Selection was started in $\mathrm{F}_{2}$.

Care had to be taken to ensure that the 20 flies of one sex chosen from a culture for assay were chosen at random with respect to the marker phenotypes segregating in the cultures. To this end the etherised flies were placed in a pile on a card, and the pile was separated into two with a needle. One-half was then collected into a pile again. The process was repeated until a sample of about 20 was obtained. This was then adjusted to precisely 20 by adding the flies lying nearest to the chosen pile or removing those nearest the discarded 
pile. Occasional counts of whole cultures indicated that this procedure did not result in selection of significant excess or deficiency of any of the major-gene phenotypes segregating in the cultures.

The experimental work in $\mathrm{F}_{1}$ and $\mathrm{F}_{2}$ was carried out by $\mathrm{D}$. S. Hathaway, then visiting Sheffield as a Fulbright Scholar. The experiment was continued thereafter by the author except that $\mathrm{Mr}$ T. B.
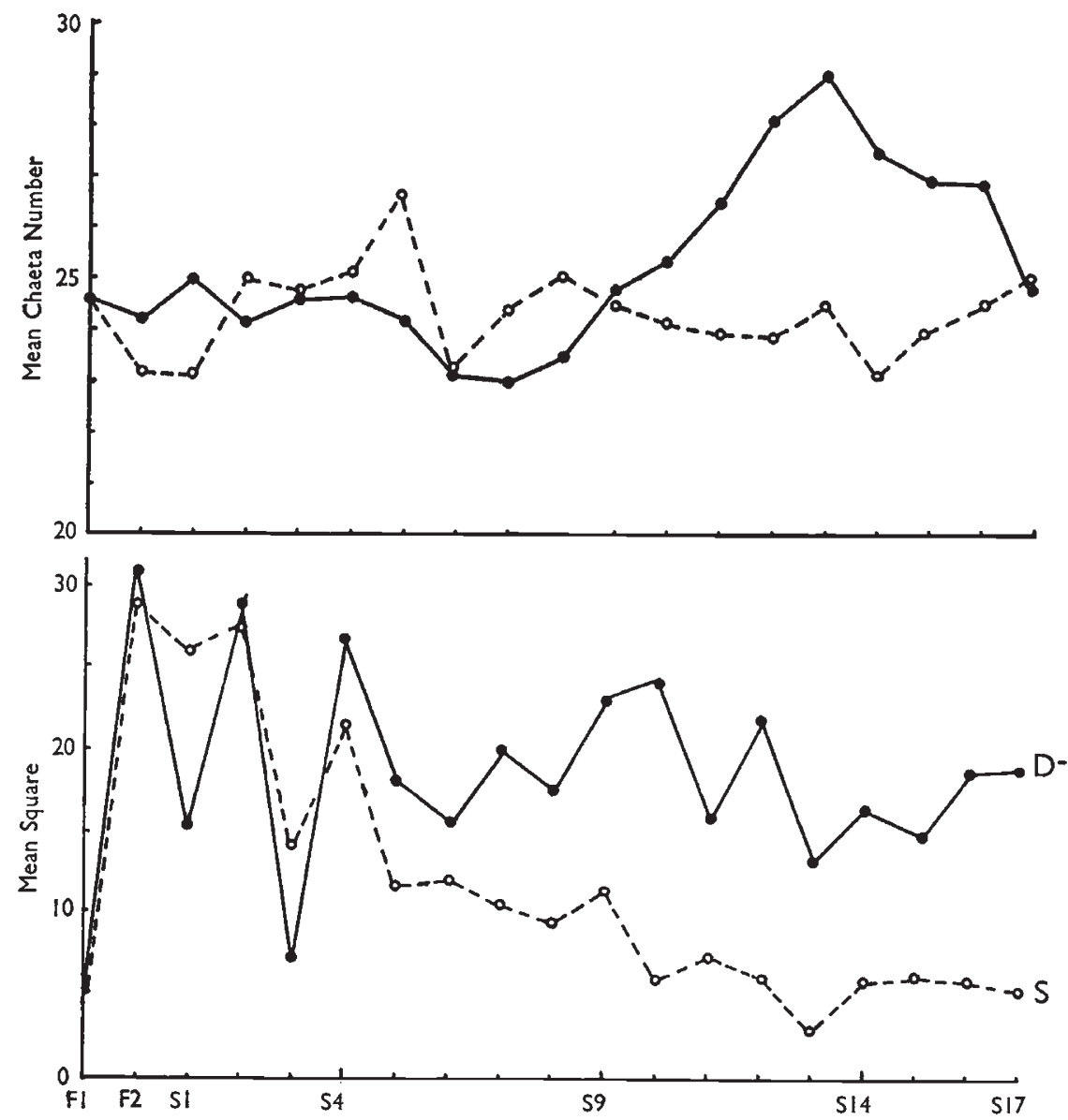

FIG. 2.-Mean chaeta numbers (upper graph) and total variance of chaeta number (lower graph for the two lines. Solid curve $\mathrm{D}^{-}$, broken curve $\mathrm{S}$. (N = I59 for each variance estimate.)

Boam maintained the lines during the absence of the author for generations $\mathrm{S}_{12}$, 13 and 14 , and did most of the virgin collection.

Serious fertility trouble in the $D^{-}$line made it necessary to end the experiment after generation $\mathrm{S}_{17}$.

\section{RESULTS}

(i) Means and variances

Fig. 2 illustrates the changes of mean chaeta number in the two lines. The only notable changes occurred in the $\mathrm{D}$ - line which for a 
period rose to a mean considerably higher than that of the $F_{1}$ or the $\mathrm{S}$ line, but at the end returned approximately to the $\mathrm{F}_{1}$ value.

Fig. 2 also illustrates the total variance of each line. The variance in $\mathrm{F}_{2}$ was very large indeed and was the same in each line. Thereafter the two lines differed.

The $S$ line in $F_{3}$ and $F_{4}\left(S_{1}\right.$ and $\left.S_{2}\right)$ maintained the high variance of $\mathrm{F}_{2}$. This was to be expected as selection of intermediates in $\mathrm{F}_{2}$ must tend to be selection for $F_{1}$ genotypes. The variance of the $S$ line fell in $\mathrm{S}_{3}$, rose again in $\mathrm{S}_{4}$, but then fell steadily to reach a level similar to that of the $F_{1}$.

The $\mathrm{D}^{-}$line, in the earlier generations of selection fluctuated widely in variance, which was also to be expected. Selection of both extremes in $\mathrm{F}_{2}$ must tend to select the parental types and negative assortative mating must then reconstitute the $F_{1}$ genotype in alternate generations. However, the fluctuations were rapidly damped out so that, from generation $\mathrm{S}_{5}$ onward, the variance of $\mathrm{D}$ - became comparatively steady, and clearly greater than that of the $\mathrm{S}$ line. The final level is midway between the variances of $F_{1}$ and $F_{2}$.

\section{(ii) The fate of the marker genes}

(a) The $S$ line. The frequencies of homozygotes for $v g, c p$ and $e$ fell rapidly in the $\mathrm{S}$ line, but the frequency of se homozygotes rose until, in generation $S_{12}$, all the flies selected were se homozygotes. $v g, \quad c p$ and $e$ did not disappear completely. $c p$ homozygotes occurred in one culture of $\mathrm{SI}_{7}, e$ homozygotes occurred in one culture of Si6, and one culture segregated $v g$ homozygotes in $\mathrm{S}_{1} 4$ though these had not been observed since $\mathrm{S}_{3}$. The chaeta numbers of these few segregants show clearly that $c p$ was still linked to low chaeta number genes and $v g$ to high, and it therefore seems likely that stabilising selection could go further, and that in doing so it might eliminate these genes completely.

Fig. 3 illustrates the relative frequencies of the sepia, clipped, sepia-clipped and ++ phenotypes amongst the non-vestigial flies of the line (clipped is hypostatic to vestigial) as far as $\mathrm{S}_{\mathrm{o}}$, after which the line was almost homozygous se. The figure shows that the initial coupling relationship of $s e$ and $c p$ was rapidly replaced in this line by corresponding repulsion products. At first these were more often $+c p$ than $s e+$, but later the frequency of $s e+$ increased greatly. Despite the few $c p$ genes that remain in the line, it is clear that stabilising selection established the se+ recombination type of chromosome as typical. Thus the line, while attaining a stable mean and relatively low variance, established in high frequency a repulsion product of the key marker genes originally put in in coupling.

(b) The $D^{-}$line. The fate of the marker genes in the $\mathrm{D}^{-}$line has been very different. The line became homozygous $v g$ in generation 

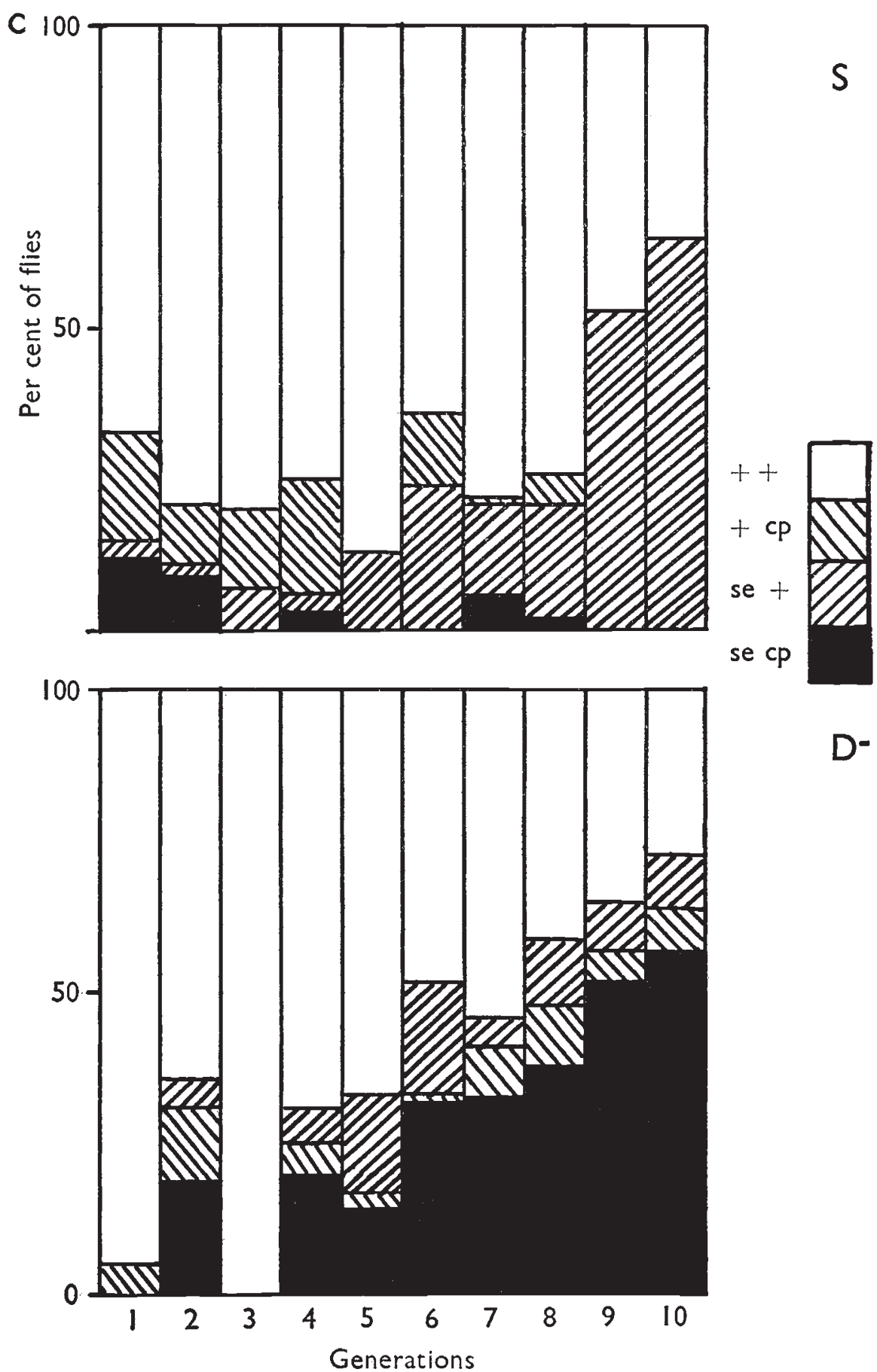

D-

FIG. 3.-Coupling and repulsion phenotypes for the markers $s e$ and $c p$ in the two lines: generations $\mathrm{S}_{1}$ to $\mathrm{S}_{\mathrm{i}}$. Black, se cp homozygotes: Hatched $+c p /+c p$ or $+c p / s e c p$, and $s e+/ s e+$ or $s e+/ s e c p$ : white, "wild-type". 
I 5, but maintained the markers $s e$ and $c p$ and their wild-type alleles in high frequency. $c p$ gives rise to difficulty as it is hypostatic to $v g$ and thus its presence could not be assessed without progeny tests after $\mathrm{S}_{\text {Io, }}$ when the line reached a high $v g$ frequency. It was, however, clear from the segregating phenotypes that $\mathrm{D}^{-}$selection favoured $v g$, se and $c p$ for the frequency of all their homozygotes rose strikingly from generation $\mathrm{S}_{3}$ to $\mathrm{S} 8$, the period in which the line was establishing the relatively stable variance roughly midway between that of $F_{1}$ and that of $\mathrm{F}_{2}$, which it maintained thereafter.

Fig. 3 demonstrates the degree to which se and $c p$ were in coupling linkage in this line. Selection increased the frequency of se cp homozygotes which became much more common than in the $\mathrm{S}$ line. It seems clear that disruptive selection tended to preserve the coupling linkage of these markers and their wild-type alleles, and in this sense its effects were the opposite of those of stabilising selection.

Because selection in the $\mathrm{D}^{-}$line picked high frequencies of $s e c p$ homozygotes as low, and of $v g$ homozygotes as high parents in each generation, many of the cultures provided test-crosses that made it possible to determine the genotypes of their parents rather completely. Until the line became largely homozygous $v g$, not only the marker genes the parents carried but also their linkage relationships could usually be determined. This was done without using the frequency relationships provided by the data which, since the samples assayed are rather small, are necessarily subject to considerable error. The conclusions concerning the locus $c p$ in the later generations have been supplemented by the results of a progeny test that was carried out on the flies available in Si 5 .

The results of this analysis are listed in table 3 which goes a long way toward explaining the history of the line. Flies homozygous for vg were often selected as high parents from the beginning, but the low parents also included $\mathrm{vg}$ homozygotes as early as $\mathrm{F}_{2}$. Such selection occurred repeatedly, and was responsible for the ultimate $v g$ homozygosity of the line.

Most of the third chromosomes selected in $\mathrm{F}_{2}$ were parental homozygotes except with respect to the $e$ locus, which is not closely linked to the known chaeta number affecting region. The flies selected in $\mathrm{S}_{\mathrm{I}}$ are all of the $\mathrm{F}_{1}$ type with respect to $s e$ and $c p$ and those selected in $\mathrm{S}_{2}$ are parental types. In $\mathrm{S}_{3} \mathrm{~F}_{1}$ types were again selected. In $\mathrm{S}_{4}$ some recombinant chromosomes were chosen, but these were not maintained. Though $s e+$ and $c p+$ chromosomes recurred, the tendency to pick up ++ chromosomes in the high flies and $s e c p$ chromosomes in the low continued. Gradually, however, ++ homozygotes disappeared, so that the high flies selected were usually $++/ s e c p$ and the low were se $c p$ homozygotes. There can be little doubt that the disruptive selection was effective in maintaining the loci $s e$ and $c p$ both heterozygous and in the coupling linkage arrangement that they had at the start of the experiment. At the end the line was maintained mainly 
TABLE 3

Genotypes of the fies selected in each generation in $D^{-}$

Female first. $\mathrm{v}=v g \quad \mathrm{~s}=s e \quad \mathrm{c}=c p \quad \mathrm{e}=e$

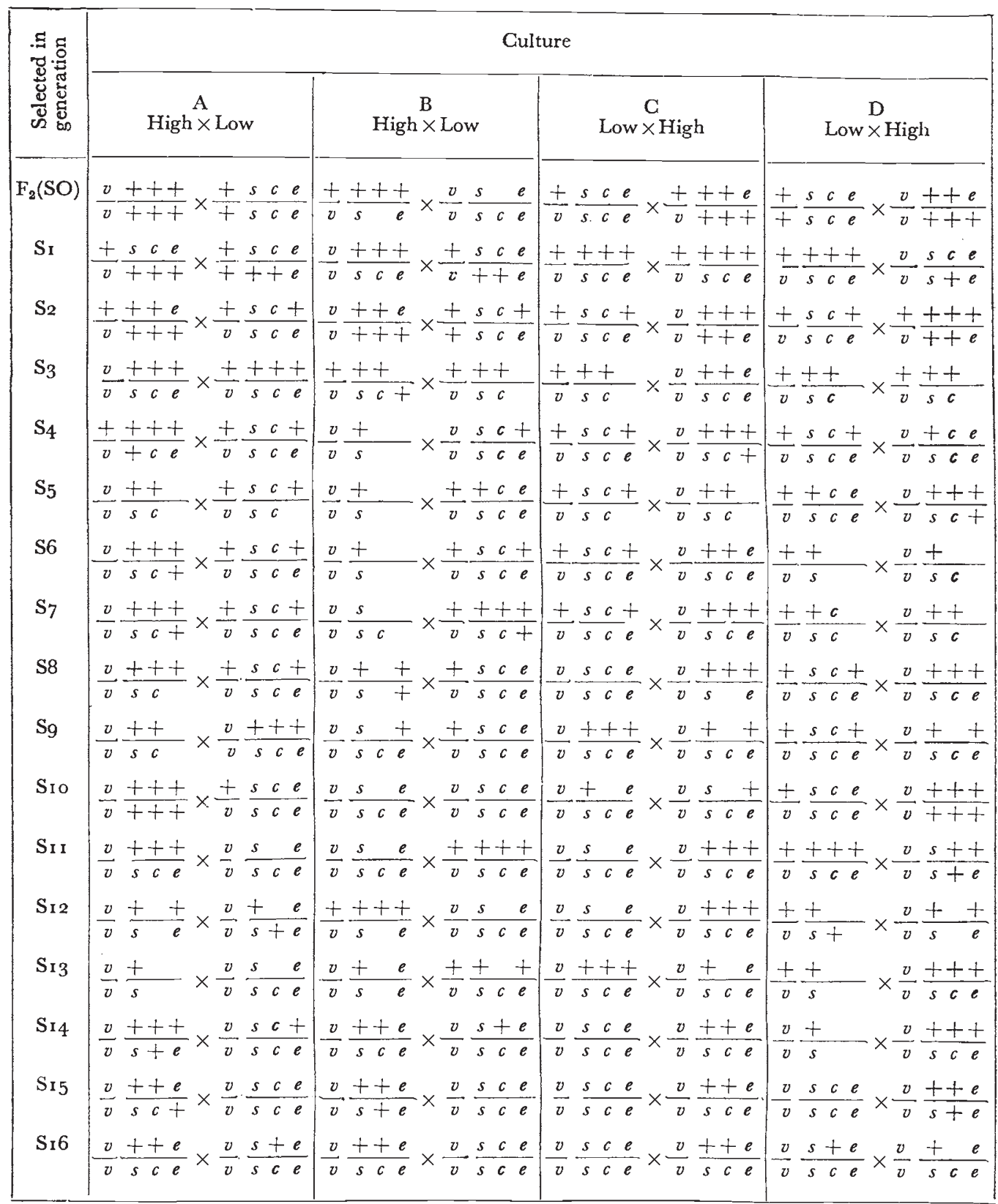

Note: Blanks indicate that it is either impossible to determine what genes the flies possessed or impossible to determine their linkage arrangements. 
by the following test-cross and its reciprocal, though there were some $s e+e$ chromosomes.

$$
\text { High } \frac{v g}{v g} \frac{++e}{\operatorname{se} e p \quad e} \times \operatorname{Low} \frac{v g}{v g} \frac{\operatorname{secpe}}{\operatorname{secpe}}
$$

(c) Recombination in the $D^{-}$cultures. In the last few generations the $\mathbf{D}^{-}$line cultures, of which there were four in each generation, fell into two classes. In any generation two of the cultures test-crossed females heterozygous for eye colour; the other two cultures testcrossed heterozygous males. Each culture segregated two classes of

TABLE 4

Means and variances in the $D^{-}$line, generations $S_{15}$ to $S_{77}$

The figure in brackets is the difference between the chaeta numbers of $+/ s e$ and se/se flies

\begin{tabular}{|c|c|c|c|c|c|c|}
\hline \multirow{3}{*}{ Gencration } & \multicolumn{3}{|c|}{ Female test-crosses } & \multicolumn{3}{|c|}{ Male test-crosses } \\
\hline & \multirow{2}{*}{$\begin{array}{l}\text { Total } \\
\text { mean } \\
\text { square }\end{array}$} & $\begin{array}{c}\text { Mean chaeta } \\
\text { numbers }\end{array}$ & \multirow{2}{*}{$\begin{array}{c}\text { Mean square } \\
\text { within } \\
\text { se classes }\end{array}$} & \multirow{2}{*}{$\begin{array}{l}\text { Total } \\
\text { mean } \\
\text { square }\end{array}$} & $\begin{array}{c}\text { Mean chaeta } \\
\text { numbers }\end{array}$ & \multirow{2}{*}{$\begin{array}{l}\text { Mean square } \\
\text { within } \\
\text { se classes }\end{array}$} \\
\hline & & $+/ s e$ se/se & & & $+/ s e \quad$ se/se & \\
\hline $\mathrm{S}_{15}$ & $8 \cdot 90$ & $\begin{array}{c}28 \cdot 6 \mathrm{I} \quad 26 \cdot 29 \\
(+2 \cdot 32)\end{array}$ & $7 \cdot 64$ & $20 \cdot 71$ & $\begin{array}{c}29 \cdot 37 \\
(+7 \cdot 36)\end{array}$ & $8 \cdot 3^{2}$ \\
\hline $\mathrm{S}_{16}$ & $14 \cdot 19$ & $\begin{array}{c}29 \cdot 53 \\
(+4 \cdot 24)\end{array}$ & $9 \cdot 77$ & $23 \cdot 61$ & $\begin{array}{c}30 \cdot 0921 \cdot 69 \\
\left(+8 \cdot 4^{0}\right)\end{array}$ & $6 \cdot 02$ \\
\hline $\mathrm{SI}_{7}$ & 17.52 & $\begin{array}{c}27 \cdot 0421 \cdot 24 \\
(+5 \cdot 80)\end{array}$ & $9 \cdot 3^{8}$ & $23 \cdot 26$ & $\begin{array}{c}28 \cdot 75 \quad 20 \cdot 72 \\
(+8 \cdot 03)\end{array}$ & $7 \cdot 04$ \\
\hline Total & 13.54 & $\begin{array}{c}28 \cdot 3924 \cdot 27 \\
(+4 \cdot 12)\end{array}$ & $8 \cdot 93$ & $22 \cdot 53$ & $\begin{array}{c}29.40 \quad 21 \cdot 47 \\
(+7.93)\end{array}$ & $7 \cdot 13$ \\
\hline
\end{tabular}

offspring distinguishable as red-eyed and sepia-eyed. As there is no crossing-over in males, comparisons of these two types of culture provide information concerning the genetic content of the chromosome types that segregated in the line. Table 4 lists the relevant data for the three generations in which $v g$ was not segregating, that is Si 5, I 6 and 17 .

The data in table 4 demonstrate that the chaeta number factors segregating in the line were complexes, not single loci, for the variance of chaeta-number in the progeny of the female test-crosses was less than that in the progeny of male test-crosses $\left(F=1 \cdot 66, N_{1}=N_{2}=237\right.$, $\mathrm{P}<\mathrm{O} \cdot \mathrm{OI})$. Such a difference seems only explicable in terms of recombination between coupled chaeta number genes, which, by producing intermediate repulsion products, would reduce the variance of the progeny of the female test-crosses.

The data also demonstrate that a significant proportion, at least, of the chaeta number factors could readily be separated from the 
locus se by recombination, for the difference of mean chaeta number distinguishing $+/$ se from se/se flies was greater in the male test-crosses than in the female (table 5). There is also evidence $(P \simeq 0 \cdot 05)$ that the variance within the se classes was greater in the female test-cross than in the male test-cross progeny, as is to be expected if the linkage between $s e^{+}$and high and between se and low chaeta number factors was readily broken by recombination.

These data therefore demonstrate that disruptive selection as practised in this line maintained coupling linkages of chaeta number genes as well as of the marker genes, despite the fact that these linkages broke down readily in each generation.

Rough linkage data obtained in the progeny test of generations $\mathrm{S}_{1} 5$ gave 27.39 per cent. recombination between $s e$ and $c p$, a value significantly higher than the standard 19.5 of Bridges and Brehme

TABLE 5

Analysis of variance of chaeta-number in the $D^{-}$line, generations $S_{I 5}$ to $S_{I 7}$

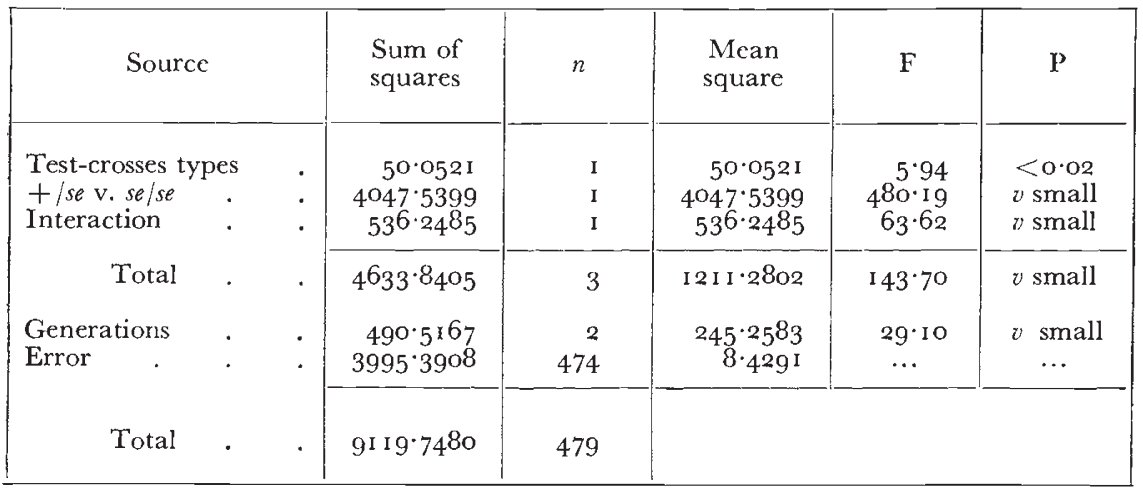

(1944). (The value for $c p-e$ was almost identical with the standard map distance.) The recombination pressure against which disruptive selection was effective was therefore considerable.

These $s e-c p$ recombination frequencies provide no evidence that selection of linked complexes of genes such as occurred in the $\mathrm{D}^{-}$line, has tightened the linkage between them. Such an effect is to be expected in appropriate circumstances (Fisher, I930), but the circumstances in the present experiment were not appropriate. Selection in the $\mathrm{D}$ - line has always been within the progeny of single females which cannot in any simple way be selection for change of recombination frequency (see Thoday and Boam, r956).

\section{DISCUSSION}

The variance that was available in the two lines at their origin was very large, and such small populations could hardly be expected to retain all of it, by whatever selection system they were maintained. Only 4 pairs of flies were selected in each generation, one out of only 
20 flies was selected, and there were 4 heterozygous marker loci. The possibility of random fixation for any of them must be considerable. The $\mathrm{F}_{2}$ chaeta-number variance was some fifteen times larger than that characteristic of wild stocks of Drosophila melanogaster, and it again might well be reduced by random fixation. It would not therefore have been surprising if the results of these experiments had been difficult to interpret.

Nevertheless the results are clear, and are consistent with the view that disruptive and stabilising selection can have very powerful effects not only on gene frequencies, but also on the frequency of coupling and repulsion phases of linkage of appropriate groups of genes as Mather's (1943) models would lead one to expect.

The stabilising selection line, though it only went to fixation at the marker locus se, nevertheless did what was to be expected of it on Mather's views. The majority of chromosomes selected were repulsion products of the key markers se and $c p$ originally put in in coupling. Taken in conjunction with the evidence of recombination of chaeta loci, and of se and chaeta loci, it seems that this repulsion product was selected for because it was a repulsion product of the chaeta loci originally put in in coupling. Whether there was good reason for the selection of $s e+$ chromosomes rather than $+c p$ chromosomes (which appeared more frequently in the earlier stages) or whether this was merely a matter of chance cannot be decided. A larger population might well have maintained both.

The disruptive selection line went to fixation for $v g$ and for the supposedly neutral locus $e$ (the latter perhaps a matter of chance), but it maintained the coupling linkages se cp and ++ with astonishing permanence, considering the frequency of recombination between these loci. The evidence presented demonstrating recombination of the chaeta number genes clearly indicates that this coupling of the markers was maintained because of their association with the chaeta number loci.

The locus vg may have become homozygous in this line by chance, but it seems unlikely, for in any normal population at $25^{\circ} \mathrm{C}$. vg is rapidly eliminated by natural selection for its normal allele. The evidence in table $\mathrm{I}$ indicates that there were interactions between the second and third chromosomes in $\mathrm{F}_{2}$, and that the difference due to the third chromosome was most effective in the presence of the second chromosomes of $v g$. It is concluded therefore that $v g$ was selected for because linked to useful modifiers that enhanced the differences of chaeta number distinguishing the $++/ s e c p$ and $s e c p / s e c p$ flies.

The difference of chaeta number effected by the se regions of chromosome III in the male test-crosses of the recent generations (table 4 ) is of the same order as that in the $\mathrm{F}_{2}$ flies that were homozygous $v g$ (table $\mathrm{I}$ ). Bearing in mind that in $\mathrm{F}_{2}$ some of the non-sepia flies will have been homozygous for the $s e^{+}$chromosome, whereas none of them can have been at $\mathrm{S}_{1} 6$ to $\mathrm{I}_{7}$ in $\mathrm{D}^{-}$, it seems clear that 
the capacity of the $v g$ chromosomes from vg 6 to enhance the effects of the $s e^{+}$chromosomes on chaeta number was exploited very fully. The $\mathrm{D}^{-}$line at the end was polymorphic; its polymorphism depended upon a complex switch "gene" in chromosome III and a genetic background functioning to enhance the effectiveness of this switch. The system is a model example of what Fisher (1930) first proposed must occur, and shows that disruptive selection can produce systems such as we expect to find and have found determining sexual dimorphism, incompatibility, mimicry and polymorphism in general.

TABLE 6

Distributions of chaeta number in the two male test-cross cultures of $D^{-}$, generation $S_{I Y}$

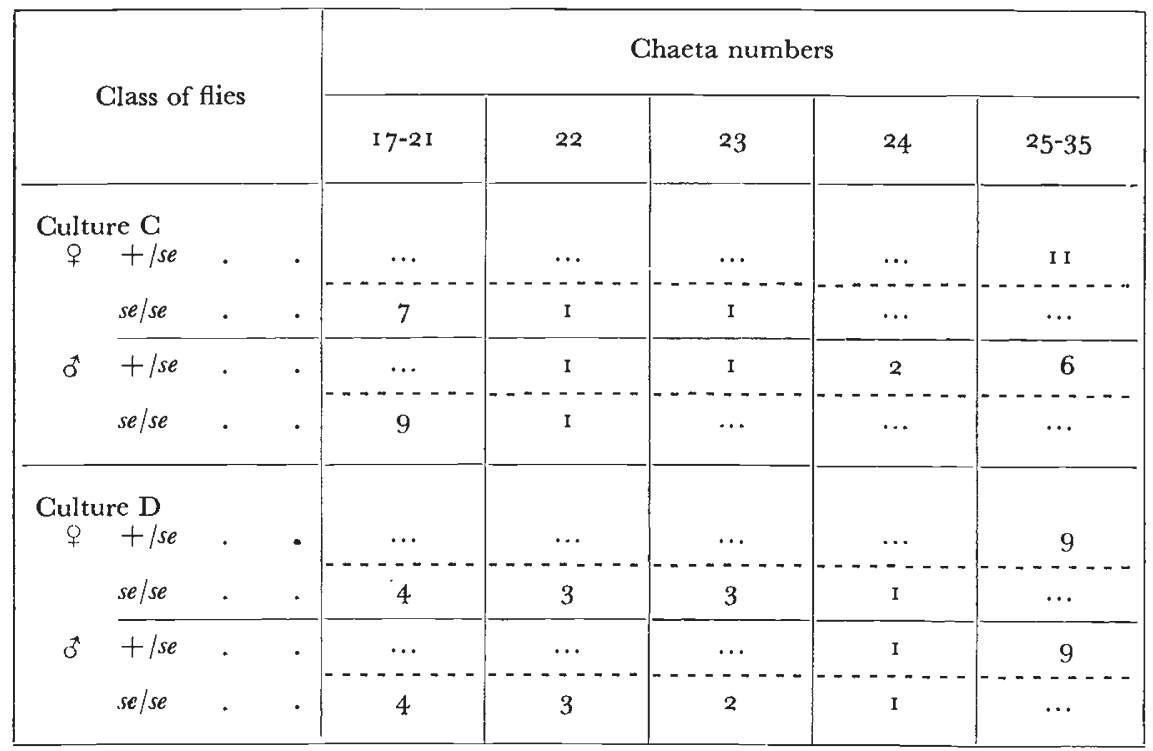

The entries give the number of flies in each class.

It may have been the same effect of the $v g$ chromosome that was responsible for its near loss in the $\mathrm{S}$ line, in which its enhancing effects on chromosome III heterogeneity would operate against the selection practised.

In the previous papers of this series it was shown that stabilising selection can reduce variance, that disruptive selection with negative assortative mating can maintain variance, and that disruptive selection with positive assortative mating can increase variance and set up a cryptic polymorphism. The present experiments confirm the reduction of variance by stabilising selection and provide strong evidence that this involves the establishment of repulsion complexes of relevant genes. They also show that the maintenance of variance by disruptive selection with negative assortative mating involves maintenance of 
coupling combinations of relevant genes. The $\mathrm{D}^{-}$line shows us further that a polymorphism can be maintained by disruptive selection with negative assortative mating. This time the polymorphism is not cryptic, but is manifest as a discontinuous morphological difference of eye colour, even though this probably depends solely on linkage of the eye colour locus to the chaeta-number genes primarily subject to selection. The line is also polymorphic for the locus $c p$, though cryptically because $c p$ is hypostatic to $v g$, and the polymorphism is manifest, though less clearly, in the key character, chaeta-number. Fig. I shows the distribution curves for generation $\mathrm{Sr}_{7}$. The $\mathrm{S}$ line distribution is very similar to that for the $F_{1}$, and does not overlap either parent distribution. The $\mathrm{D}^{-}$-distribution overlaps both parental distributions and is clearly bimodal. This bimodality is strikingly associated with the distribution of the eye colour markers. In fact, within any sex and culture of the two male test-cross cultures of that generation, there is very little overlap of the chaeta-numbers of red-eyed and sepia-eyed flies (table 6). When recombination is prevented, therefore, the polymorphism is almost discontinuous even in chaeta-number alone.

These experiments therefore confirm the results of those published earlier in showing that disruptive selection can be responsible for polymorphism and they extend them in demonstrating that coupling arrangements of linked genes, and background genotypes enhancing the effects of these switching gene complexes are involved. There seems little reason to doubt that similar and more spectacular results must readily occur in nature where much larger populations and longer times are available.

\section{SUMMARY}

I. One population (S) has been exposed to stabilising selection and another $\left(\mathrm{D}^{-}\right)$to disruptive selection with negative assortative mating, each for I 7 generations.

2. The two populations were established from the $F_{1}$ of a high sternopleural chaeta-number line (vg 6) homozygous for $v g$, and a low chaeta-number line homozygous for $s e c p$ and $e$. It was known that a considerable part of the genetic material responsible for the high chaetanumber of $\mathrm{vg} 6$ was in the $s e-c p$ region of chromosome III. The $\mathrm{F}_{2}$ data showed that the effect of these genes was enhanced in the presence of the $v g$ chromosome II of $v g$. The variance of chaeta-number in $\mathrm{F}_{2}$ was very large indeed.

3. Stabilising selection reduced variance greatly. Disruptive selection maintained a very high variance.

4. Stabilising selection reduced the frequency of the vg marker on chromosome II to a low level and presumably also the linked enhancers. It established se $c p^{+}$repulsion chromosomes at high frequency.

5. The disruptive selection line became homozygous for the vg marker, but remained heterozygous for the markers se and $c p$. These 
markers and their + alleles were kept in the coupling phase of linkage despite considerable recombination pressure.

6. It could be shown that recombination of chaeta-number genes also was occurring in the $\mathrm{D}^{-}$line, so that disruptive selection must have been eliminating the repulsion products. This was clearly the cause of the maintenance of the markers in coupling.

7. The disruptive selection line was polymorphic for the locus se, and cryptically polymorphic for $c p$ (which is hypostatic to $v g$ ) at the end of the experiment. It was also polymorphic for chaeta-number, this polymorphism depending on a switching gene complex in chromosome III with its effect enhanced by the modifiers in chromosome II.

8. The experiments confirm that disruptive selection can maintain a polymorphism, and show that coupling linkages of appropriate genes are involved. They also show that stabilising selection can function by establishing repulsion linkages of genes hitherto in the coupling phase.

\section{REFERENCES}

BRIDGES, G. S., AND BREHME, K. 1944. The mutants of Drosophila melanogaster. Carnegie Institute of Washington Publication $55^{2}$.

FAlconer, D. S. 1958. Selection for phenotypic intermediates in Drosophila. $\mathcal{F}$. Genet., 55, 55 I-561.

FISHER, R. A. I930. The Genetical Theory of Natural Selection. Oxford. LERNER, I. M. 1958. The Genetic Basis of Selection. Wiley, New York.

MATHER, K. 1943. Polygenic balance and natural selection. Biol. Rev., 18, 32-64. MATHER, K. I953. The genetical structure of populations. Symp. Soc. Exp. Biol., $7,66-95$.

MATHER, K. 1955. Polymorphism as an outcome of disruptive selection. Evolution, $9,52-6 \mathrm{r}$.

simpson, G. G. 1944. Tempo and Mode in Evolution. Columbia, New York.

THODAY, J. M. 1958. Effects of disruptive selection : the experimental production of a polymorphic population. Nature, I8I, I I 24-I 125 .

THODAY, J. м. 1959. Effects of disruptive selection. I. Genetic flexibility. Heredity, 13, $187-203$.

THODAY, J. M., AND BOAM, т. в. 1956. A possible effect of the cytoplasm on recombination in Drosophila melanogaster. F. Genet., 54, 456-46r.

Thoday, J. M., AND BOAM, т. в. 1959. Effects of disruptive selection. II. Polymorphism and divergence without isolation. Heredity, 13, 205-218.

waddington, c. H. 1953. Epigenetics and evolution. Symp. Soc. Exp. Biol., 7, r86-r99.

waddington, c. H. 1958. The Strategy of the Genes. Allen \& Unwin, London. 